\title{
BIBECHANA
}

A Multidisciplinary Journal of Science, Technology and Mathematics

ISSN 2091-0762 (online)

Journal homepage: http://nepjol.info/index.php/BIBECHANA

\section{On the critical points of a polynomial}

\author{
N A Rather, Faroz Ahmad* \\ Department of Mathematics, University of Kashmir \\ *Corresponding author: Email: farozmaths1080@gmail.com \\ Article history: Received 25 March, 2012; Accepted 13 August, 2012
}

\begin{abstract}
Let $P_{n}$ denote the set of all polynomials of the form $\quad P(z)=(z-a) \prod_{j=1}^{n-1}\left(z-z_{j}\right)$ with $\quad|a| \leq 1$ and $\left|z_{j}\right| \geq 1,1 \leq j \leq n-1$. In this paper, we show that $P^{\prime}(Z) \neq 0$ in $\left|z-\left(\frac{n-1}{n}\right) a\right|<\frac{1}{n}$ for all polynomials $P \in P_{n}$. For $a=\mathbf{0}$, this reduces to a result due to Aziz and Zargar.
\end{abstract}

Keywords: Polynomial, Critical point; Sendove conjecture; Walish Coincidence theorem.

\section{Introduction}

The Gauss-Lucas Theorem states that if $\mathrm{S}$ is the set of zeros of a polynomial

$$
P(z)=\prod_{j=1}^{n}\left(z-z_{j}\right)
$$

then every zero of the derivative $P^{\prime}(z)$ is contained in the smallest convex set that contains $\mathrm{S}$. This is best possible, in the sense that, if $P(z)$ has all its zeros in $D=\{z:|z| \leq 1\}$, then no proper subset of $D$ can be guaranteed to contain even one zero of $P^{r}(z)$, (as is shown by the polynomial of the form $P(z)=(z-a)^{n}$, since $\quad P^{x}(z)=n(z-a)^{n-1}$ has zeros only at $a$, which can lie anywhere in $D$ ). Gauss-Lucas theorem has been rather thoroughly investigated [8] and sharped in several ways. However, there is one related question that deserves attention, namely given one specific zero $Z_{n}$ of $P(Z)$, what can be said about a neighborhood of $Z_{n}$ that will always contain a zero of $P^{x}(z)$. 
The following conjecture was made by Bulgarian Mathematician B L Sendov in 1962 but became later known as Ilief's conjecture (See [4, problem 4.5] or [6, p.795]) .

Conjecture 1. Let $P(z)$ be a polynomial of degree $n$ having all its zeros in the unit disk $|Z| \leq 1$. If $\boldsymbol{a}$ is any one of these zeros, then $P^{x}(\boldsymbol{z})$ has at least one zero in the disk $|z-a| \mathbf{5} \mathbf{1}$. Since in 1962, when it is first became known, Conjecture 1 has been the subject of more than thirty articles. However, it was fully verified only for polynomials of degree $n \leq \mathbf{8}$ (see [13]). A variety of special cases have been dealt with over the years (See $[2,7,11]$ for references), among which we mention that of a polynomial with at most five distinct zeros [5], as well as Miller's qualitative result [10], according to which those zeros of $P(z)$ lying sufficiently close to the unit circle satisfy an even stronger condition that the one stated in Sendov's conjecture (See also [12]).

Another Stronger conjecture than that of Ilief was made in 1969 by Goodman, Rahman and Ratti [3].

Conjecture 2. Let $P(z)$ be a polynomial of degree $n$ having all its zeros in the unit disk $|z| \leq \mathbf{1}$. If $a$ is any one of these zeros, then $P^{r}(z)$ has at least one zero in the disk

$$
\left|z-\frac{a}{2}\right| \leq 1-\frac{|\alpha|}{2} \text {. }
$$

Conjecture 2 has been proved when $|a|=1 \quad$ [3], but some counter examples have been devised for case $|a|<1$ by M. J. Miller [10].

Recently Aziz and Zargar [2] have proved the following result .

Theorem A. If $P(z)=z \prod_{j=1}^{n-1}\left(z-z_{j}\right)$ is a polynomial of degree $n$ with $\left|z_{j}\right| \geq 1$, $j=1,2, \cdots, n-1$, then $P^{r}(z)$ does not vanish in $|z|<\frac{1}{n}$.

In this paper we establish a generalized form of above theorem. In fact we prove the following interesting result which extracts that portion of complex plane in which the above polynomial does not vanish.

Theorem 1. Let

$$
P(z)=(z-a) \prod_{j=1}^{n-1}\left(z-z_{j}\right)
$$

be a polynomial of degree $n$ with $|a| \leq 1$ and $\left|z_{j}\right| \geq \mathbf{1}, j=1,2, \cdots, n-1$, then $P^{x}(z)$ does not vanish in the disk 


$$
\left|z-\left(\frac{n-1}{n}\right) a\right|<\frac{1}{n}
$$

The result is best possible as shown by the polynomial $P(z)=(z-a)\left(z-e^{i \alpha}\right)^{n-1}$, $0 \leq \alpha<2 \pi$. Further taking $a=0$ we get Theorem A. By using a similar argument, we can prove the following more general result.

Theorem 2. Let

$$
P(z)=(z-a)^{k} \prod_{j=1}^{n-k}\left(z-z_{j}\right)
$$

be a polynomial of degree $n$ with $|a| \leq 1$ and $\left|z_{j}\right| \geq 1, j=1,2, \cdots, n-k$ where $1 \leq k \leq n-1$. Then $P^{\prime}(z)$ has $k-1$ fold zero at $z=a$ and remaining $n-k$ zeros of $P^{\prime}(z)$ lie in the region $\left|z-\left(\frac{n-k}{n}\right) a\right| \geq \frac{k}{n}$. The result is best possible as shown by the polynomial

$$
P(z)=(z-a)^{k}\left(z-e^{i \alpha}\right)^{n-k}, \quad 0 \leq \alpha<2 \pi .
$$

For the proof of this theorem we need the following lemma which is the coincidence theorem of Walish [8, P.62] (see also [1]).

Lemma. Let $G\left(Z_{1}, Z_{2}, \ldots, Z_{n}\right)$ be a symmetric $n$-linear form of total degree $n$ in $Z_{1}, Z_{2}, \ldots, Z_{n}$ and let $\mathrm{C}$ be a circular region containing the $n$ points $w_{1}, w_{2}, \ldots, w_{n}$, then there exists at least one point $\alpha$ belonging to $\mathrm{C}$ such that

$$
G(\alpha, \alpha, \ldots, \alpha)=G\left(w_{1}, w_{z}, \ldots, w_{n}\right) .
$$

Proof of theorem 2. By hypothesis,

$$
P(z)=(z-a)^{k} \prod_{j=1}^{n-k}\left(z-z_{j}\right)
$$

where $|a| \leq 1$ and $\left|z_{j}\right| \geq 1, \quad j=1,2, \cdots, n-1 . \quad$ Let $T(z)=\prod_{j=1}^{n-k}\left(z-z_{j}\right)$, then $T(z)$ is a polynomial of degree $n-k$, having all its zeros in $|z| \geq 1$ and we have

$$
P(z)=(z-a)^{k} T(z) \text {. }
$$

This implies

$$
P^{x}(z)=k(z-a)^{k-1} T(z)+(z-a)^{k} T^{x}(z)
$$


If now $W$ is any zero of $P^{\prime}(z)$, then from $(1)$, we get

$$
k(w-a)^{k-1} T(w)+(w-a)^{k} T^{r}(w)=P^{\prime}(w)=0 .
$$

This is an equation which is linear and symmetric in the zeros of $T(z)$ that is, in $Z_{1}, Z_{2}, \ldots, Z_{n-1}$. Hence an application of the above lemma with circular region $\quad C=\{z:|z| \geq 1\}$ shows that $W$ will also satisfy the equation obtained by substituting into the equation (2)

$$
T(z)=(z-\alpha)^{n-k}
$$

where $\alpha$ is suitably chosen point in the circular region $\{Z:|z| \geq 1\}$. That is $W$ satisfies the equation

$$
\text { or equivalently } \begin{aligned}
& k(w-a)^{k-1}(w-\alpha)^{n-k}+(w-a)^{k}(n-k)(w-\alpha)^{n-k-1}=\mathbf{0} \\
& (w-a)^{k-1}(w-\alpha)^{n-k-1}(n w-(n-k) a-k \alpha)=0 .
\end{aligned}
$$

Thus $w$ has the values $w=\alpha \quad$ or $w,=\left(\frac{n-k}{n}\right) a+\frac{k \alpha}{n} \quad$ where $\alpha$ is suitably chosen point in $\{z:|z| \geq \mathbf{1}\}$. if $w=\alpha$, then using the fact that $|a| \leq \mathbf{1}$, it follows that

$$
\begin{aligned}
\left|w-\left(\frac{n-k}{n}\right)\right| a \mid & =\left|\alpha-\left(\frac{n-k}{n}\right)\right| a \mid \\
\geq|\alpha|-\left(\frac{n-k}{n}\right)|a| & \\
& \geq 1-\left(\frac{n-k}{n}\right)|a| \\
& \geq 1-\left(\frac{n-k}{n}\right) \\
& =\frac{k}{n} .
\end{aligned}
$$

If

then clearly

$$
w=\left(\frac{n-k}{n}\right) a+\frac{k \alpha}{n}
$$

Thus in any case

$$
\left|w-\left(\frac{n-k}{n}\right) a\right|=\frac{k|\alpha|}{n} \geq \frac{k}{n}
$$

$$
\left|w-\left(\frac{n-k}{n}\right) a\right| \geq \frac{k}{n}
$$

Since $W$ is an arbitrary zero of $P^{s}(z)$, it follows that every zero of $P^{s}(z)$ lie in the disk

This completes the proof of Theorem 2 .

$$
\left|w-\left(\frac{m-k}{n}\right) a\right| \geq \frac{k}{n} \text {. }
$$

Corollary . If we take $k=\mathbf{1}$ in Theorem 2, we get Theorem 1 . 
N. A. Rather and Faroz Ahmad / BIBECHANA 9 (2013) 28-32: BMHSS, p.32 (Online Publication: Nov., 2012)

\section{References}

[1] A. Aziz, Pacific J. Math., 118 (1985) 17-26.

[2] Aziz and Zargar, Aus. Math. Soc., 57 (1998) 173-174.

[3] A. W. Goodman, Q. I. Rahman and J. S. Ratti, Proc. Amer. Math . Soc., 21 (1969) 273-274.

[4] W. K. Hayman, Research Problems in Function Theory, Athlone Press London (1967).

[5] S. Kumar and B. G. Shenoy, J. Math. Anal. Appl., 171 (1992) 595-600.

[6] M. Marden, Much ado about nothing, Amer. Math. Monthly, 83 (1976)788-789.

[7] M. Marden, Amer. Math. Monthly, 90 (1983) 267-276.

[8] M. Marden, Geometry of polynomials, IInd edition Math. Surveys, 3. Amer .Math. Soc., Providence, Rhode Island (1966).

[9] A. Meir and A. Sharma, Pacific J. Math., 31 (1969) 459-467.

[10] M. J. Miller, J. Math. Anal. Appl., 175 (1993) 632-639.

[11] G. Schmeisser, Math. Z., 156 (1977) 165-173.

[12] V.Vajaitu and Z. Zaharesen, London. Math. Soc., 25 (1993) 49-54.

[13] Jonny E. Brown and Guangping Xiang, proof of the Sendov conjecture for polynomials of degree at most eight (1965)

[14] Z. Rubinstien, Pacific J. Math., 26 (1968) 159-161. 